\title{
MODIFICACIÓN DE CARACTERES LIGADOS A LA DOMESTICACIÓN EN CUCURBITA MAXIMA. UTILIZACIÓN DE LA MORFOMETRÍA COMO HERRAMIENTA PARA SU IDENTIFICACIÓN
}

\author{
Analía B. MARTÍNEZ ${ }^{1 *}$; Sergio I. PEREZ ${ }^{2,5 ;}$ Verónica S. LEMA ${ }^{3,5}$ \\ y Fernando LÓPEZ ANIDO ${ }^{4}$
}

\author{
${ }^{1}$ Infive, Universidad Nacional de La Plata. Diag 113 y 61. La Plata. Argentina \\ ${ }^{2}$ División Antropología, Facultad de Ciencias Naturales y Museo, Universidad Nacional de La Plata. \\ Paseo del Bosque s/n. La Plata, Argentina \\ ${ }^{3}$ Laboratorio de Etnobotánica y Botánica Aplicada. División Arqueología, Facultad de Ciencias Naturales \\ y Museo, Universidad Nacional de La Plata. Paseo del Bosque s/n. La Plata, Argentina \\ ${ }^{4}$ Facultad de Ciencias Agrarias. Universidad Nacional de Rosario. Zavalla. Argentina \\ ${ }^{5}$ Consejo Nacional de Investigaciones Científicas y Técnicas \\ *Autor para correspondencia: anas_martinez@hotmail.com
}

\begin{abstract}
RESUMEN. Modificación de caracteres ligados a la domesticación en Cucurbita maxima. Utilización de la morfometría como herramienta para su identificación. El objetivo de este trabajo consiste en reconocer patrones de variación morfométrica -mediante la aplicación de análisis multivariado para tamaño y formaen semillas de C. maxima que sean diagnósticos para formas domesticadas, silvestres e híbridas actuales, a fin de aplicarlos a la identificación de semillas arqueológicas. Para lograr este objetivo se midieron con calibre digital 1317 semillas de las formas antedichas y de nueve sitios arqueológicos de Argentina y Perú. Se exploró el patrón de variación entre las mismas en tamaño y forma mediante análisis multivariado. Se identificó una tendencia general hacia la disminución de la variabilidad en forma y tamaño a lo largo del tiempo, con una primera etapa donde se mantuvieron ejemplares híbridos, generándose nuevas formas y una segunda donde se mantuvieron los rasgos de momentos previos y el aumento del tamaño de las semillas.
\end{abstract}

Palabras clave. Cucurbita, domesticación, silvestre, híbrido

SUMMARY. Modification of characters linked to Cucurbita maxima domestication. Using morphometry as a tool for identification. The aim of this paper is to recognize patterns of morphometric variation -through the application of multivariate analysis for size and shape- in C. maxima seeds, which are diagnostic for current domesticated, wild and hybrid forms, in order to be applied to the identification of archaeological seeds. In order to achieve this aim, 1317 seeds were measured with digital caliber corresponding to the former forms and to nine archaeological sites from Argentina and Peru; patterns of variation among them were explored in both size and shape, employing multivariate analysis. A general trend in time towards diminution of variability in size and shape was identified with a first stage in which hybrid forms were maintained and new shapes were generated and a second stage in which features of previous moments were maintained and the size of the seeds were increased.

Key words. Cucurbita, domestication, wild, hybrid. 


\section{INTRODUCCIÓN}

La selección artificial y la manipulación de individuos o poblaciones vegetales en función de las preferencias que las sociedades humanas tienen sobre ellas, ha provocado la variación de caracteres morfológicos y fisiológicos, entre otros, en diversas especies. Este tipo de prácticas puede dar lugar a poblaciones cultivadas y/o domesticadas. Estas últimas se caracterizan por una total dependencia de las plantas respecto del Hombre en las distintas etapas de su ciclo de vida (Casas et al. 1999).

El género Cucurbita (familia Cucurbitaceae, subfamilia Cucurbitoideae) es estrictamente americano e incluye una serie variable de especies tanto silvestres como domesticadas cuyo número varía entre doce y veintiséis de acuerdo con las diferentes taxonomías que se tomen en cuenta (Whitaker \& Bemis, 1976,Jeffrey, 1980, Nee, 1990, Lira Saade, 1995, Bisognin, 2002). Dado que la familia es esencialmente tropical, sus miembros se han adaptado bien a climas templados con baja incidencia de heladas, aunque se han desarrollado cultivares con tolerancia a bajas temperaturas y con capacidad para crecer rápidamente en suelos fríos (Whitaker, 1974). Todas ellas presentan frutos de tipo pepo, cuyas formas, tamaños y colores son muy variables. Son plantas de hábito mayormente herbáceo, con tallos rastreros y con fecundación cruzada (Whitaker, 1964). Es un género utilizado desde tiempos prehispánicos, con una gran cantidad de restos arqueológicos desde México a Argentina. Los hallazgos más tempranos (ca. 9000-8000 AP) se ubican entre la costa norte de Perú y la costa de Ecuador (Dillehay et al. 2007, Stothert et al. 2003). Las especies de este género son consideradas entre las primeras en el proceso de domesticación (Cutler, 1961) y poseen usos alimenticios, medicinales, ornamentales y para hacer artefactos.

En Sudamérica las especies domesticadas son Cucurbita moschata (Duch ex Lam) Duch. ex Poir., Cucurbita maxima subsp. maxima Duch. ex Lam y Cucurbita ficifolia Bouché. Existiendo también en esta área formas espontáneas: Cucurbita maxima Duch. ex Lam. subsp. andreana (Naudin) Filov y Cucurbita ecuadorensis Cutler \& Whitaker.

C. maxima subsp. maxima se distribuye actualmente en gran parte de Sudamérica, es sensible a las heladas, y la más tolerante a las bajas temperaturas luego de C. ficifolia; puede tener hábito de enredadera o arbusto (C. maxima subsp. maxima cv. zapallito como arbustiva). Su fruto puede presentar una forma de "turbante" cuando el ovario queda por fuera del receptáculo y posee gran número de cultivares (Whitaker, 1974, Lira Saade, 1995, Bisognin, 2002).

C. maxima subsp. andreana es una hierba anual muy variable en tamaño y de tallos rastreros (Ashworth \& Galetto, 1999). Esta subespecie es muy propensa a variar, sobre todo en el tamaño de los frutos, los cuales pueden ser ovoides, oblongos, esféricos o deprimidos; su longitud varía de 6 a 21,5 cm (Millán, 1945, Martínez Crovetto, 1974, Ashworth, 1997). La mayoría de las poblaciones actuales de este taxón se ubican en el centro-este de Argentina y Uruguay (Nee, 1990, Lira Saade, 1995), aunque recientemente se han encontrado poblaciones en Bolivia (Andres \& Nee, 2005) y Perú (Valega Rosas et al. 2004). En cuanto a sus relaciones evolutivas, gran cantidad de autores (Whitaker \& Bemis, 1964), (Nee, 1990, Decker-Walters \& Walters 2000, Sanjur et al. 2002) coinciden en señalar a subsp. andreana como el antecesor de subsp maxima. Si bien estos autores proponen la zona comprendida entre el sur de Perú-Bolivia Noroeste argentino (NOA) como área de origen, la evidencia arqueológica no lo corrobora aún. Esto se debe a que es posible encontrar en los mismos contextos arqueológicos, restos de la subespecie silvestre junto a la domesticada y a formas que comparten caracteres entre ambas subespecies (Lema, 2009a).

Indagar sobre relaciones evolutivas, de 
origen y domesticación a través de la morfología de semillas actuales y arqueológicas en el género Cucurbita, cuenta con bastos antecedentes para los miembros norteamericanos del género y muy pocos para aquellos de Sudamérica (Lema, 2009a). Generalmente se ha recurrido al empleo de medidas lineales tales como largo, ancho y espesor de las semillas para diferenciar formas silvestres de domesticadas (Decker \& Wilson, 1986, Cowan \& Smith, 1993, Newsom et al. 1993, Cowan, 1997, Smith 2000, 2006), asumiéndose que existe una tendencia a que las semillas aumenten de tamaño a través de la evolución bajo domesticación. En el caso particular de Cucurbita spp. se planteó que el aumento en el tamaño (largo) podía ser el resultado de la selección cultural dirigida, hacia semillas más grandes dado que las mismas eran consumidas (Decker \& Wilson, 1986), o bien que semillas más grandes están indirectamente reflejando la selección de frutos de mayor tamaño, considerando una correlación entre tamaño de semilla y de fruto (Cowan, 1997). En este sentido Bisognin (2002) invierte los términos, afirmando que la selección de semillas más grandes pudo haber resultado en frutos más grandes. Semillas más grandes poseen embriones de mayor tamaño, lo cual es una ventaja adaptativa para la germinación en medios antrópicos (Decker \& Wilson, 1986).

En términos generales, la etapa inicial de domesticación estaría caracterizada por una tendencia a la disminución en el coeficiente de variación $(\mathrm{CV})$ respecto de las poblaciones silvestres, luego este tenderá a aumentar por la coexistencia de formas silvestres y otras que exhiben rasgos novedosos, malezoides e híbridos, y con el tiempo la variabilidad disminuiría por la presencia de fuertes presiones selectivas ligadas a la domesticación. Pero con la generación de diversos cultivares, la diversidad aumentaría nuevamente a nivel específico, si bien debido a que los cultivares tenderán a ser muy uniformes en sus características, la variabilidad intrínseca de los mismos será baja (Gremillion, 1993). Cowan \& Smith (1993) registran un CV de entre 5,4 para largo y 6,6 para ancho en semillas de una población de morfotipo silvestre de $C$. pepo L. Para estos y otros autores (Gremillion, 1993, Newsom et al. 1993) estos valores de CV bajos indican homogeneidad morfológica. Estos valores aumentarán con la domesticación por la presencia de más de un tipo de fruto y/o los efectos iniciales de la hibridación, cultivo y cría selectiva (Newsom, 1993). Por ende, un aumento en el $\mathrm{CV}$ o un $\mathrm{CV}$ alto en conjuntos arqueológicos puede indicar la presencia de un taxón domesticado con variedades de frutos de morfología muy diferente (Cowan \& Smith, 1993). La persistencia de una alta variabilidad a lo largo de una secuencia arqueológica señala además que ciertas prácticas tales como el aislamiento reproductivo y la cría selectiva estaban limitadas en frecuencia o grado (Gremillion, 1993). El análisis de cultivares de ssp. maxima ha indicado que estas tendencias no siempre se dan, ya que ciertos cultivares locales pueden tener valores de CV elevados; además el empleo de medidas lineales, índices, CV y análisis de conglomerado, no reflejan las clasificaciones y agrupamientos locales de variedades de zapallo (Lema, 2009 b).

Cuando las medidas de los ejemplares arqueológicos poseen un valor intermedio al registrado entre poblaciones silvestres y domesticadas, o bien cuando el rango de medidas de ejemplares arqueológicos abarca el de ambas poblaciones actuales, se interpreta que se está ante formas malezoides resultado de la hibridación entre poblaciones silvestres y domesticadas (Gremillion, 1993) o ante formas transicionales en un continuum de transformaciones (Pearsall, 1989, Hillman \& Davies, 1990). El primero de los casos mencionados es el que hasta ahora se considera, en relación a los complejos subsp. maxima -subsp. andreana en el registro arqueológico del NOA, a partir de análisis uni y bivariados a nivel morfológico de semillas y a nivel 
anatómico en las testas de las mismas (Lema, 2009a).

Varios autores consideran que en poblaciones vegetales silvestres la forma es menos variable y está menos sujeta a factores ambientales que el tamaño, por lo cual es más adecuado el uso de índices que el de medidas lineales (Mangafa \& Kotsakis, 1996). Esto se aplicaría particularmente a las semillas ya que en las mismas la forma es un rasgo genético más conservador que el tamaño (Kay et al. 1980). Los análisis morfométricos realizados en poblaciones actuales de diversas especies demuestran que este tipo de aproximación resulta ser apropiada para determinar patrones de similitud o de variación morfológica en frutos y semillas procedentes de poblaciones sujetas a diversas prácticas de manejo cuando se aplican análisis multivariados (Colunga García, 1984, Casas \& Caballero, 1996, Casas et al. 1999, Casas, 2001, Aguirre-Dugua et al. 2012, 2013, Rodríguez Morales et al. 2013).
Estudios previos en el género constatan una tendencia al aumento del tamaño de las semillas, sin embargo, la ausencia de estudios morfométricos en poblaciones híbridas generadas bajo cruzamientos controlados, dificulta el avance en la comprensión de la historia evolutiva del taxa, quedando ésta enmarcada en dos grandes esferas (silvestre - domesticado), sin que se analice con mayor detalle los procesos de hibridación que pudieron existir a lo largo del tiempo. Este último aspecto es sobre el que se centra este trabajo, considerando que hasta el presente sólo se cuenta con ejemplares arqueológicos de la forma domesticada en restos de semillas, estando la forma silvestre representada sólo por restos arqueológicos de pericarpio (Lema, 2009a).

El objetivo de este trabajo consiste en reconocer patrones de variación morfométrica -mediante la aplicación de análisis multivariado para tamaño y forma- en semillas de C. maxima

\begin{tabular}{|c|c|c|c|c|c|}
\hline Taxa & $\begin{array}{c}\text { Código } \\
\text { UNR }\end{array}$ & Fuente* & $\begin{array}{l}\text { Código Banco } \\
\text { Germoplasma }\end{array}$ & $\begin{array}{l}\text { Nombre } \\
\text { común }\end{array}$ & Origen \\
\hline $\begin{array}{l}\text { C. maxima } \\
\text { ssp. maxima }\end{array}$ & 56 & GRIN & PI 234608 & $\begin{array}{c}\text { Queensland } \\
\text { Blue }\end{array}$ & South Africa \\
\hline $\begin{array}{l}\text { C. maxima } \\
\text { ssp. maxima }\end{array}$ & 93 & $\begin{array}{l}\text { Feltrin } \\
\text { Sementes }\end{array}$ & - & $\begin{array}{l}\text { Bobrincha } \\
\text { de tronco } \\
\text { Redonda } \\
\text { Verde }\end{array}$ & Brasil \\
\hline $\begin{array}{l}\text { C. maxima } \\
\text { ssp. maxima }\end{array}$ & 153 & $\begin{array}{l}\text { Semillas } \\
\text { CAPS }\end{array}$ & - & $\begin{array}{c}\text { Zapallo Gris } \\
\text { Plomo }\end{array}$ & Argentina \\
\hline $\begin{array}{c}\text { C. maxima } \\
\text { ssp. andreana }\end{array}$ & 130 & IPK & MAX 81 & $\begin{array}{c}\text { ssp. } \\
\text { andreana }\end{array}$ & $\begin{array}{l}\text { Argentina (Entre } \\
\text { Ríos) }\end{array}$ \\
\hline $\begin{array}{c}\text { C. maxima } \\
\text { ssp. andreana }\end{array}$ & 140 & $\begin{array}{c}\text { Colector } \\
\text { Pablo López } \\
\text { Anido }\end{array}$ & UNR 140 & & $\begin{array}{c}\text { Argentina } \\
\text { (Santiago del } \\
\text { Estero) }\end{array}$ \\
\hline $\begin{array}{c}\text { C. maxima } \\
\text { ssp. andreana }\end{array}$ & 160 & GRIN & G 29254 & $\begin{array}{c}\text { ssp. } \\
\text { andreana }\end{array}$ & $\begin{array}{l}\text { Argentina } \\
\text { (Córdoba) }\end{array}$ \\
\hline
\end{tabular}

Tabla 1. Accesiones parentales empleadas en los cruces experimentales, parental accesión involved in the experimental crosses, GRIN, Germplasm Resources Information Network USA (http://www.ars-grin. gov),Feltrin Sementes, Brazil (http://www.sementesfeltrin.com.br/produtos-categoria-abobrinha), Semillas CAPS (Compañía Argentina Productora de Semillas) (http://www.semillascaps.com.ar), IPK Leibniz Institute of Plant Genetics and Crop Plant Research, Gatersleben, Germany (http://www.ipk-gatersleben.de/Internet). 


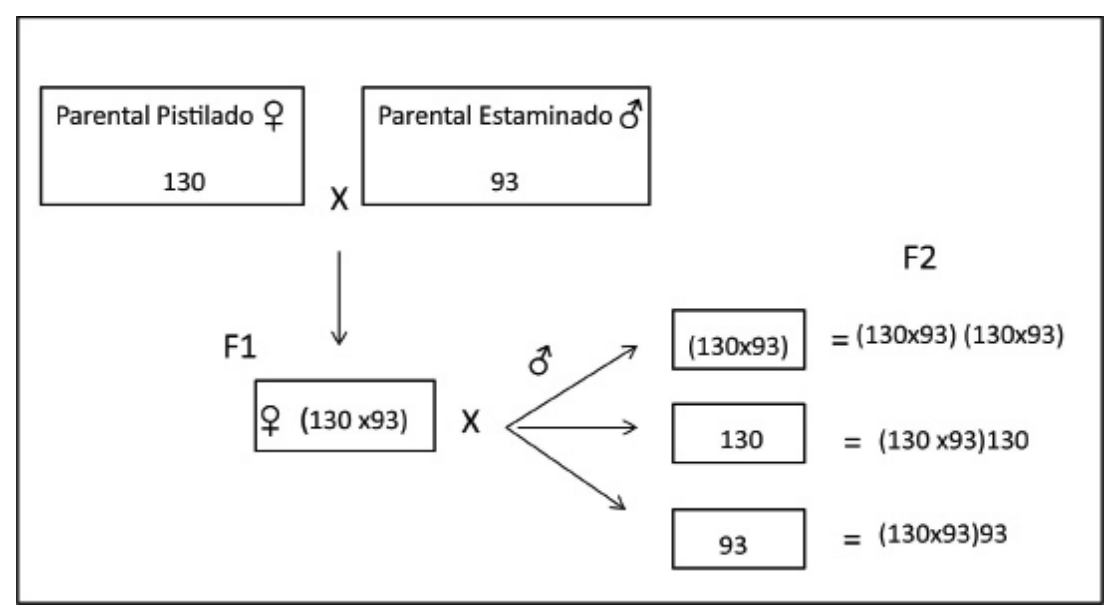

Figura 1: Gráfico que representa a modo de ejemplo, un cruce experimental realizado entre accesiones de la subespecie silvestre (130) y la subespecie domesticada (93).Posteriormente se obtiene la primer generación -F1- (130 x 93).En este caso a las plantas femeninas (híbridas) se las cruza con formas masculinas silvestres (130) ó domesticadas (93) o inclusive con plantas de genotipo similar al femenino (Back Cross) obteniendo así la generación F2. Graph showing, by way of example, an experimental cross made between the wild subspecies accessions (130) and the domesticated subspecies (93). Subsequently, -F1- the first generation (130 x 93) is obtained. In this case, female plants (hybrid) are crossed with wild male forms (130) or domesticated ones (93) or even with plants with a similar genotype to female genotype (Back Cross), thus obtaining F2 generation

que sean diagnósticos para formas domesticadas, silvestres e híbridas actuales, a fin de aplicarlos a la identificación de semillas arqueológicas. Este objetivo se enmarca en los intereses de un proyecto de investigación multidisciplinario enfocado en la reconstrucción de la historia de la domesticación del zapallo criollo, C. maxima subsp. maxima, a partir de su antecesor silvestre (subsp. andreana). Las aproximaciones llevadas a cabo en este proyecto han sido cuatro: el cultivo experimental de ambas subespecies y la producción controlada de híbridos, ensayos fisiológicos sobre las generaciones obtenidas, estudios morfométricos sobre semillas actuales de ambas subespecies e híbridos y el estudio de restos vegetales recuperados en sitios arqueológicos de Argentina y Perú.

\section{MATERIALES Y MÉTODOS}

Las semillas analizadas corresponden a tres grandes grupos:

1) Cruces experimentales. Material vegetal actual de las subespecies C. maxima subsp. maxima, C. maxima subsp. andreana y cruces entre ambas (generaciones F1 y F2), fueron generados en el campo experimental Villarino de la Facultad de Ciencias Agrarias de la Universidad Nacional de Rosario (Zavalla, Argentina) bajo condiciones controladas durante los años 2012, 2013 y 2014. Se emplearon tres accesiones por cada parental (tab. 1).A modo de ejemplo se indica la orientación de los cruces controlados (fig. 1).

La generación F1 consistió en cruces que involucraron a ambas especies como parental pistilado; la generación F2 incluye cruces entre F1 o de F1 con alguno de sus parentales.

2) Colección de referencia. Material vegetal actual de las subespecies maxima y andreana. En el caso de la primera se incluyen tres cultivares comerciales (Inglés, Criollo y Hubbard) cedidos por la Cátedra de Horticultura (Facultad de 


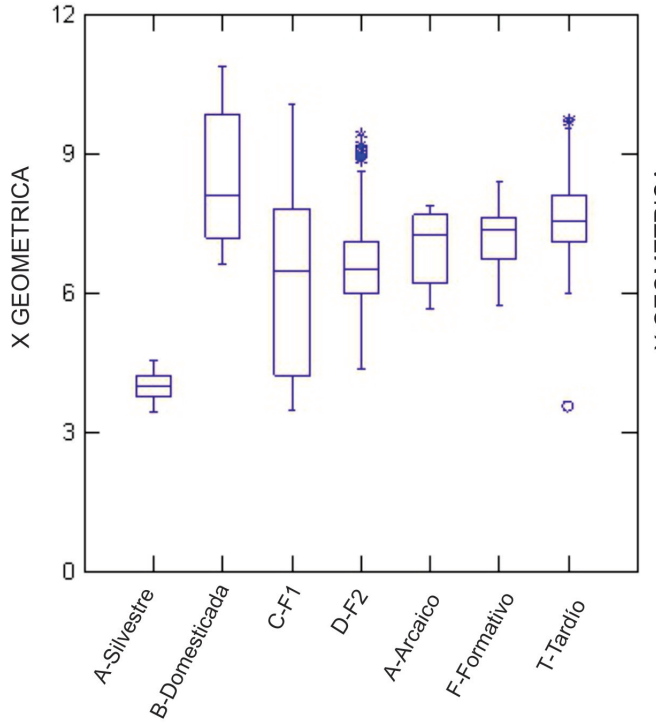

GENOTIPOS

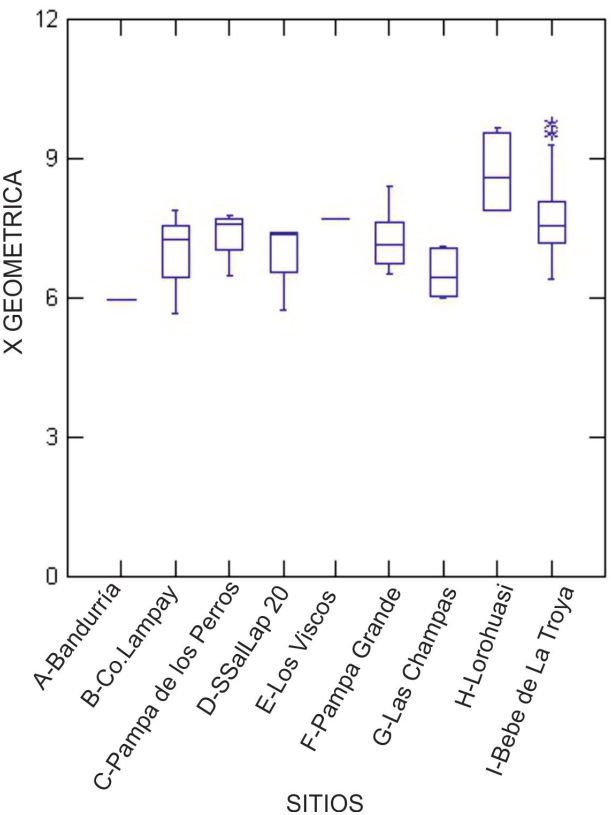

Figura 2. Media geométrica. A) Los genotipos silvestres y domesticados (A y B) incluyen colección de referencia más parentales de cruzamientos experimentales, los restos arqueobotánicos se ordenan temporalmente (E-G); B) semillas arqueológicas analizadas, de izquierda a derecha se ordenan los sitios de más tempranos a más tardíos. Geometric mean A) Wild and domesticated genotypes (A and B), reference collections and parents of experimental crosses included; archeobotanics remains arrenged chronologically (E-G); B) Analyzed archeological seeds, sources sites sorted from earliest to latest.

Ciencias Agrarias, Universidad Nacional de La Plata) y cinco cultivares locales obtenidos durante trabajo de campo en el NOA (Lema, 2009a y b): zapallito, boliviano, tronquero, y dos provenientes de los Valles de Salta. En el caso de la segunda subespecie se cuenta con ejemplares cedidos por la investigadora L. Ashworth que corresponden a tres poblaciones obtenidas por polinización cruzada, autogama y libre en el centro de Argentina.

3) Restos arqueobotánicos. Material vegetal arqueológico que corresponde a semillas de C. maxima desecadas procedentes de sitios arqueológicos del NOA y del centro-sur de Perú. Entre los primeros se cuenta con ejemplares de los períodos Formativo o Temprano (ca. 2000-1200 AP; sitios: SSalLap 20, Pampa Grande y Los Viscos) y Tardío (ca. 1200-800 AP; sitios: Bebe de la Troya, Las
Champas y Lorohuasi). Entre los segundos se cuenta con ejemplares de los sitios Bandurria, Cerro Lampay y Pampa de los Perros que corresponden al período Arcaico (ca. 3000-2000 AP).

Para poder caracterizar morfométricamente la naturaleza del material se midieron con calibre digital el largo, ancho y espesor de cada semilla $(\mathrm{N}=1317$; grupo $1 \mathrm{~N}=835$; grupo $2 \mathrm{~N}=$ 349; grupo $3 \mathrm{~N}=133$ ). Se empleó análisis de Componentes Principales (CP), que estima ejes multivariados de mayor variación dentro de la muestra, sobre estas tres variables para conocer y dimensionar la variabilidad de los conjuntos analizados, identificando qué variable puede aportar mayor información al conocimiento de la variación entre las semillas y grupos estudiados. La variación total del conjunto de datos fue particionada en un componente de 
Analysis of Variance

\begin{tabular}{|c|c|c|c|c|c|}
\hline Source & Type III S S & Df & Mean Square & F-ratio & p-value \\
\hline VAR_1\$ & 964.223 & 6 & 160.704 & 96.779 & 0 \\
\hline Error & 1592.443 & 959 & 1.661 & & \\
\hline
\end{tabular}

Tukey's Honestly-Significant-Difference Test

\begin{tabular}{|c|c|c|c|c|c|}
\hline \multirow[t]{2}{*}{ VAR_1S (i) } & \multirow[t]{2}{*}{ VAR_1\$ (i) } & \multirow[t]{2}{*}{ Difference } & \multirow[t]{2}{*}{ p-value } & \multicolumn{2}{|c|}{$95.0 \%$ Confidence Interval } \\
\hline & & & & Lower & Upper \\
\hline A-Silvestre & B-domesticada & -4.426 & 0 & -5.096 & -3.756 \\
\hline A-Silvestre & C-F1 & -2.012 & 0 & 2.465 & -1.56 \\
\hline A-Silvestre & D-F2 & -2.571 & 0 & -3.018 & -2.125 \\
\hline A-Silvestre & E-Arcaico & -2.937 & 0 & -4.428 & -1.447 \\
\hline A-Silvestre & F-Formativo & -3.232 & 0 & -4.36 & -2.105 \\
\hline A-Silvestre & G-Tardío & -3.634 & 0 & -4.172 & -3.096 \\
\hline B-Domesticada & C-F1 & 2.414 & 0 & 1.837 & 2.991 \\
\hline B-Domesticada & D-F2 & 1.855 & 0 & 1.283 & 2.428 \\
\hline B-Domesticada & E-Arcaico & 1.489 & 0.064 & -0.044 & 3.022 \\
\hline B-Domesticada & F-Formativo & 1.194 & 0.046 & 0.011 & 2.377 \\
\hline B-Domesticada & G-Tardío & 0.792 & 0.006 & 0.146 & 1.438 \\
\hline $\mathrm{C}-\mathrm{F} 1$ & D-F2 & -0.559 & 0 & -0.848 & -0.27 \\
\hline $\mathrm{C}-\mathrm{F} 1$ & E-Arcaico & -0.925 & 0.494 & -2.377 & 0.526 \\
\hline $\mathrm{C}-\mathrm{F} 1$ & F-Formativo & -1.22 & 0.014 & -2.295 & -0.145 \\
\hline $\mathrm{C}-\mathrm{F} 1$ & G-Tardío & -1.622 & 0 & -2.038 & -1.205 \\
\hline D-F2 & E-Arcaico & -0.366 & 0.99 & -1.816 & 1.083 \\
\hline D-F2 & F-Formativo & -0.661 & 0.536 & -1.733 & 0.411 \\
\hline D-F2 & G-Tardío & -1.063 & 0 & -1.473 & -0.653 \\
\hline E-Arcaico & F-Formativo & -0.295 & 0.999 & -2.076 & 1.486 \\
\hline E-Arcaico & G-Tardío & -0.697 & 0.809 & -2.177 & 0.784 \\
\hline F-Formativo & G-Tardío & -0.402 & 0.939 & -1.515 & 0.712 \\
\hline
\end{tabular}

Figura 3: Analisis Estadístico Anova y Tukey. Statistic analysis.Anova and Tukey 


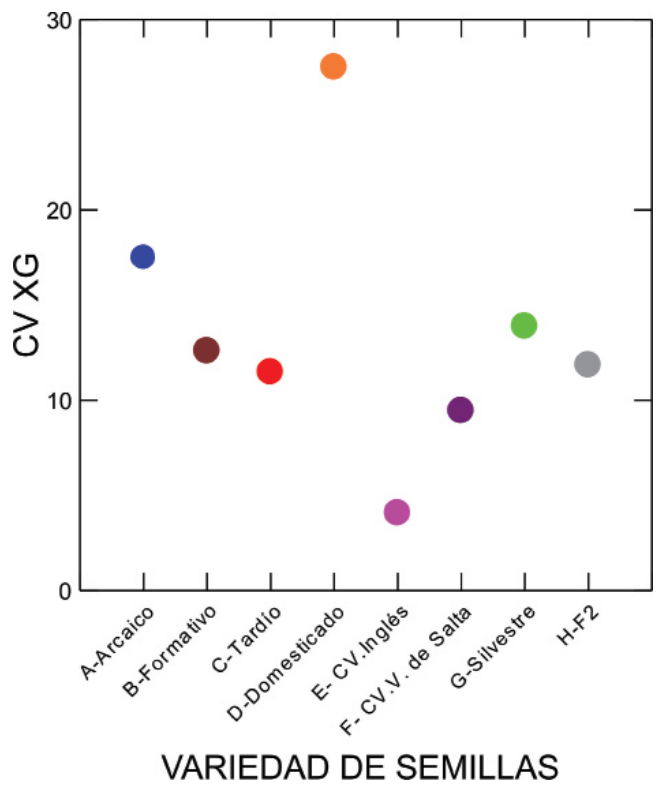

Figura 4. Coeficiente de variación de la media geométrica. Se señalan los conjuntos de semillas arqueológicas de cronología diversa (A-C), cultivares de referencia con los valores menores (Inglés) y mayores (Valles de Salta) registrados dentro del conjunto analizado, todos los genotipos silvestres (G) y domesticados (D) (colección de referencia más parentales de cruzamientos experimentales) y F2 generados experimentalmente $(\mathrm{H})$.

Azul: Arcaico; Naranja: Domestiacado; Verde: Silvestre ; Marrón: Formativo; Rosa: CV. Ingles; Gris: F2; Rojo: Tardío; Violeta: CV. V Salta. Variation coefficient of the geometric mean. Archeological seeds from diverse chronology $(A-C)$; reference cultivars with the lowest (Inglés) and highest values (Valles de Salta) among the analyzed group, all wild $(G)$ and domesticated genotypes $(D)$ (reference collection plus parents of experimental crosses) and generated F2 (H). Blue: Archaic; Orange: Domestiacad; Green: Silvestre; Brown: Formative; Pink: CV. English; Gray: F2; Red: Late; Violet: CV.V Salta

tamaño y otro de forma. Se estimó el tamaño de cada semilla como la media geométrica (media aritmética de las variables originales en escala logarítmica; Jungers et al. 1995) del largo, ancho y espesor y la forma de las mismas mediante el índice largo/ancho (Corruccini,
1995, Jungers et al. 1995). Debido a que el alto de las semillas no aportaba variación considerable dentro de la muestra, esta medida no fue utilizada en los análisis de forma (ver más abajo).

\section{RESULTADOS Y DISCUSIÓN}

\section{Tamaño}

La media geométrica del tamaño (fig.2 A), permitió diferenciar poblaciones silvestres y domesticadas. Las semillas pertenecientes a la generación F1 y F2 ocuparían un rango de variación intermedio entre las anteriores, principalmente F2. El material arqueobotánico de los períodos Arcaico y Formativo tiene un rango de distribución coincidente con F2 (híbridos), F1 y con la forma domesticada, en tanto el momento tardío coincide mayormente con la forma domesticada y en menor medida con F1 y F2. La presencia de hibridación y generación de complejos silvestre-cultivadodomesticado en C. maxima durante momentos formativos en el NOA ya había sido planteada por una de las autoras de este trabajo (Lema, 2009a). Con los análisis aquí presentados esta idea se reforzaría y se haría extensiva a momentos arcaicos y -de manera más acotada a algunos sitios- durante el Tardío. Resultado que también se corrobora con el análisis estadístico que muestra claras semejanzas entre las formas arqueológicas y las formas actuales (fig. 3).

El análisis de las semillas arqueológicas (fig. 2 B) indica una tendencia hacía el aumento de tamaño de las mismas a lo largo del tiempo, desde momentos arcaicos a tardíos. Esta tendencia, sin embargo, no es uniforme ya que en el caso del sitio tardío Las Champas, el tamaño de las semillas es notablemente menor al de los sitios precedentes y contemporáneos. Asimismo, parte de la variación del sitio tardío Bebe de la Troya coincide con lo registrado para momentos cronológicos previos. En este último sitio, análisis anatómicos en testas de estas 
semillas arqueológicas sugerían hibridación (Ratto et al. 2014). El conjunto de semillas de Lorohuasi es el que presenta mayor diferencia respecto a las restantes muestras arqueológicas, esto puede deberse a que el sitio corresponde a momentos de ocupación inkaica o posterior a la conquista española (Ratto et al. 2010).

La mayor proporción de las semillas medidas muestra valores para el $\mathrm{CV}$ de la media geométrica entre 10 y 15 (fig. 4). Considerando este indicador, durante el Arcaico, la variabilidad de tamaños es mayor a la del resto de los genotipos, salvo lo registrado en formas domesticadas actuales (D), conjunto que exhibe gran variabilidad debido a que en la muestra se han incluido once cultivares. Hacía el período Formativo y Tardío esta variabilidad disminuye -con mínima variación entre ambos- asemejándose al valor registrado en F2. Ninguno de los conjuntos arqueológicos posee variabilidad semejante a los cultivares actuales de referencia, tanto a los menos (cultivar comercial Inglés- D) como a los más variables (cultivar local Valles de Salta-D), por lo cual puede pensarse en la presencia de más de un cultivar en los conjuntos arqueológicos agrupados cronológicamente. Así y todo puede afirmarse que hubo una tendencia a la disminución de la variabilidad de tamaños a lo largo del tiempo.

\section{Forma}

Dado que el análisis de componentes principales no arrojó un resultado significativo para la variable alto, ésta no fue empleada para los análisis de forma. La varianza explicada para cada variable fue de: Largo $97 \%$, Ancho $1,34 \%$ y el alto $0,72 \%$. El índice largo/ancho no resultó útil para distinguir los distintos genotipos actuales entre sí. Pero sí, para diferenciar éstos de los conjuntos arqueológicos (fig. 5A). La figura 5B muestra

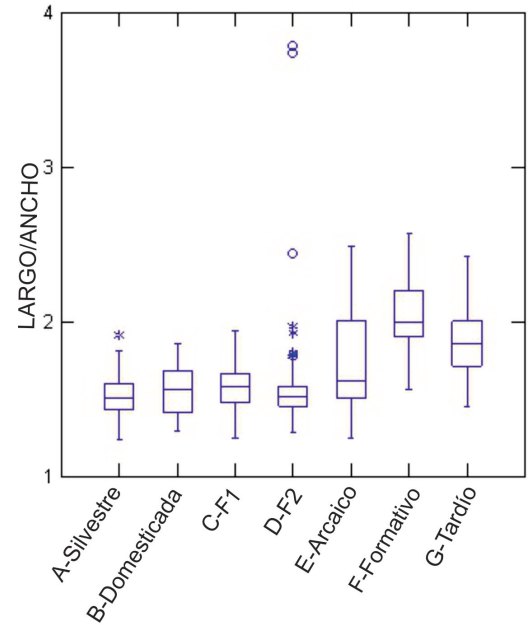

GENOTIPOS

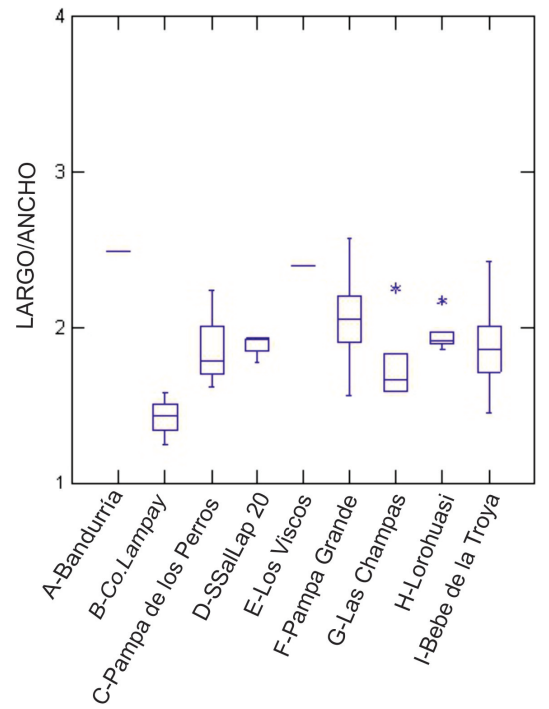

SITIOS

Figura 5. Relación largo/ancho. A: se señala en todos los genotipos silvestres y domesticados (colección de referencia, más parentales de cruzamientos experimentales), F1, F2 y material arqueobotánico (E-G). B: conjuntos de semillas arqueológicas desde las más tempranas a la izquierda a las más tardías a la derecha. Lenght/width ratio. A: Wild and domesticated genotypes (reference collection plus parents of experimental crosses), F1, F2 and archeobotanic materials (E-G). B: From left to right, earliest and latest archeological seeds sites. 


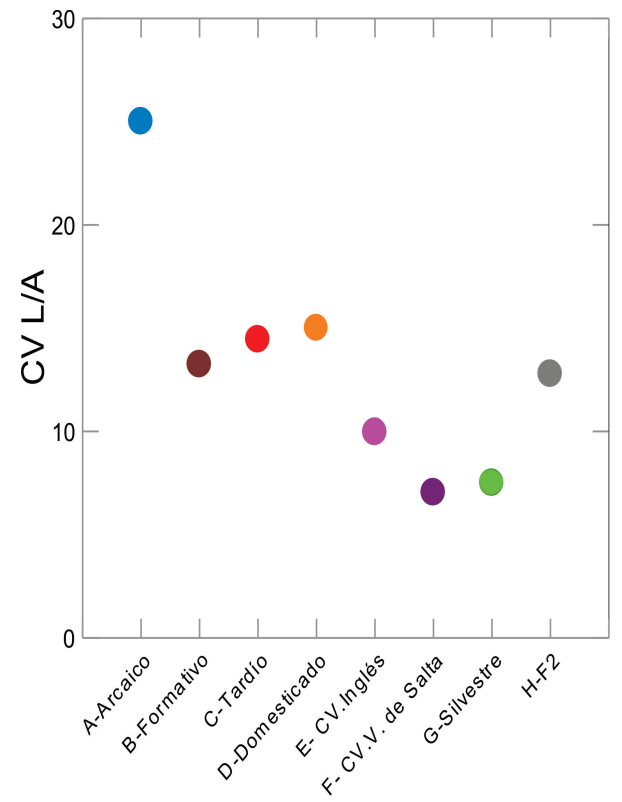

VARIEDAD DE SEMILLAS

Figura 6. Coeficiente de variación para la forma (relación largo/ancho). Se señalan los conjuntos de semillas arqueológicas de cronología diversa (A-C), cultivares de referencia con los valores menores (Valles de Salta) y mayores (Inglés) registrados dentro del conjunto analizado, todos los genotipos silvestres y domesticados (colección de referencia más parentales de cruzamientos experimentales) y F2 (D-H). Azul: Arcaico; Naranja: Domestiacado; Verde: Silvestre; Marrón: Formativo; Rosa: CV. Ingles; Gris: F2; Rojo: Tardío; Violeta: CV.V Salta. Seed shape (Lenght/width ratio) coefficient of variation. Archeological seeds from diverse chronology $(A-C)$; reference cultivars with the lowest (Valles de Salta) and highest values (Inglés) among the analyzed group, all wild and domesticated genotypes (reference collection plus parents of experimental crosses) and generated $F 2(D-H)$.

Blue: Archaic; Orange: Domestiacad; Green: Silvestre; Brown: Formative; Pink: CV English; Gray: F2; Red: Late; Violet: CV. V Salta

una gran variabilidad de formas para el Arcaico, siendo esta variabilidad menor y semejante entre conjuntos del Formativo y el Tardío. Durante el Formativo habría habido formas de semillas donde predomina el largo, exclusivas de este momento, mientras que para el Tardío la relación largo/ancho está nuevamente comprendida dentro de las registradas para el Arcaico.

Si bien puede decirse que entre los conjuntos de semillas arqueológicas existe una tendencia a lo largo del tiempo hacia formas donde prepondera el largo (fig. 5B), al igual que en el caso del tamaño, esta tendencia no se mantiene totalmente durante el Tardío. El rango de distribución de formas de este último período ya está presente en momentos previos.

En lo que refiere al CV de la forma (fig. 6), las semillas de sitios arqueológicos tempranos presentan un valor mayor respecto de los demás valores registrados, en este caso es incluso mayor al de las formas domesticadas. Hacia el período Formativo disminuye la variabilidad de formas (semejante a F2) y se incrementa levemente hacia el Tardío, siendo semejante al CV registrado para formas domesticadas.

\section{CONCLUSIONES}

El material vegetal generado experimentalmente fue importante para caracterizar los conjuntos de restos arqueobotánicos de distinta cronología. A diferencia del índice largo/ancho, la media geométrica resultó ser un indicador útil para diferenciar poblaciones silvestres, domesticadas, F1 y F2. Su aplicación a restos arqueobotánicos indica que durante momentos arcaicos y formativos habría tamaños que coinciden con formas híbridas, a diferencia de momentos tardíos, salvo para los restos del sitio Las Champas. Esto quizá es el motivo por el cual los conjuntos de semillas de ambos períodos tempranos poseen un $\mathrm{CV}$ de tamaño mayor. En cuanto a la forma, los conjuntos arqueológicos -salvo parte del Arcaico- no coinciden con las registradas en ejemplares actuales.

A lo largo del tiempo, puede verse que entre el período Arcaico y Formativo no hay cambios notables de tamaño, pero sí de forma, con semillas más largas en este último. Durante 
el Tardío sí hay semillas de tamaño mayor, junto a otras de tamaño y forma igual a las de momentos previos. No se registran formas nuevas durante el Tardío.

En cuanto a la variabilidad de los conjuntos arqueobotánicos, ésta es mayor en tamaño y forma durante el Arcaico, luego disminuye para ambos rasgos durante el Formativo y también para el Tardío, aunque en este último caso aumenta levemente la variabilidad de formas al incluir la registrada en ambos momentos cronológicos previos.

Resumiendo, puede decirse que la tendencia general luego de haberse domesticado $C$. maxima subsp maxima fue, primero, la generación de nuevas formas, manteniéndose poblaciones híbridas, lo cual no aportó mayor variabilidad de forma o tamaño ya que la misma se vio reducida durante el Formativo. En momentos posteriores, continuó la reducción de la variabilidad, se mantuvieron las mismas formas y tamaños, a la vez que se optó por sumar a los conjuntos tamaños mayores y se redujo-si bien no completamente- la presencia de formas híbridas.

Los análisis presentados en este trabajo evidencian que el estudio de la forma y el tamaño de manera conjunta, resultan útiles para dilucidar tendencias en las prácticas de manejo de poblaciones vegetales en el pasado que no son lineales u homogéneas. La continuidad en esta clase de estudios resulta esencial para conocer y ponderar el manejo de la diversidad biocultural de poblaciones cultivadas y domesticadas desde una perspectiva de largo plazo.

\section{BIBLIOGRAFÍA}

ANDRES, T. \& M. NEE -2005- The Goldman Cucurbit collecting Expedition in southern Bolivia and Northwestern Argentina. WWW. cucurbit.org

ANSHWORTH, L.-1997- Estudios sobre la biología reproductiva del zapalllo amargo (Cucurbita andreana, Cucurbitaceae). Tesina de Licenciatura. F.C.E.F y N. U.N.N.
ANSHWORTH, L. \& L. GALETTO -1999Morfoanatomía cuantitativa de las flores estaminadas y pístiladas de Cucurbita maxima subsp andreana (Cucurbitaceae). Darwiniana 37 (3-4):187 198.

BISOGNIN, D. -2002- Origin and evolution of cultivated Cucurbits. Ciencia Rural.VOL 32 ISSN 0103-8478

CASAS, A., J. CABALLERO, A. VALIENTE BANUET, A. SORIANO \& P. DAVILA -1999- Morphological Variation and process of Domestication of Stenocereus Stellatus (Cactaceae) in Central Mexico. American Journal of Botany 86(4): 522-533.

CORRUCCINI, R.S. -1995- Of ratios and rationality. Am J Phys Anthropol 96:189-191.

COWAN, C.W. \& B.D. SMITH -1993- New perspectives on a wild gourd in Eastern North America. Jounal of Ethnobiology 13(1):17-54

COWAN, C. -1997- Evolutionary changes associated with the domestication of Cucurbita pepo En: K. Gremillion (Ed.) People, plants and landscapes. Studies in Paleoethnobotany pp: 63-85. University of Alabama Press.

CUTLER, H. \& T. WHITAKER -1961- History and distribution of cultivated cucurbits in the Americas American Antiquity 26:469-485

DECKER, D. \& H. WILSON -1986- Numerical analysis of seed morphology in Cucurbita pepo. Systematic Botany (1986) 11 (4): 595-607

DECKER-WALTERS, D. \& T. WALTERS -2000Squash. The Cambridge World History of food Vol.1 K.F. Kiple, K.C. Ornelas (Eds.) Cambridge University Press.

DILLEHAY, T., J. ROSSEN, T. ANDRES \& D. WILLIAMS -2007- Preceramic adoption of peanuts, squash and cotton in Northern Peru. Science 316: 1890-1893.

GREMILLION, K. -1993- The evolution of seed morphology in domesticated Chenopodium: an archaeological case study. Journal of Ethnobiology 13(21): 149-169

HILLMAN, G. \& M. S. DAVIES -1990- Measured domestication rates in wild wheats and barley under primitive cultivation, and their archaeological implications. Journal of World Prehistory 4 (2): 157-222.

JEFFREY, C. -1980-A review of the Cucurbitaceae Botanical Journal of the Linnean society 81:233-247 
JUNGERS, W.L., A. FALSETTI \& C.E. WALL -1995- Shape, relative size and size-adjustments in morphometrics. Yrbk Phys Anthropol 38:137-161.

KAY, S., A. KING, \& M. ROBINSON -1980- Study of archaeobotanical seeds. American Antiquity $35: 26-39$.

LEMA, V.- 2009a - Domesticación Vegetal y grado de dependencia ser humano-planta en el desarrollo Cultural Prehispánico del Noroeste Argentino. Tesis doctoral. UNLP

LEMA, V. -2009b-Criterios de selección en los procesos de manipulación vegetal: el potencial de la información etnobotánica en la interpretación de restos arqueobotánicos de Cucurbita sp. Darwiniana 47 (1): 35-55

LIRA SAADE, R. -1995- Estudios taxonómicos y ecogeográficos de las Cucurbitaceae latinoamericanas de importancia económica. Instituto de Biología, UNAM, México.

MANGAFA, M. \& K. KOTSAKIS -1996- A new method for the identification of wild and cultivated charred grape seeds. Journal of archaeological science 23: 409-418

MARTÍNEZ CROVETO, R .-1974- Cucurbitaceae. En A Burkart (ed) Flora ilustrada de la provincia de Entre Ríos. Colección Científica Instituto Nacional de Tecnología Agropecuaría 4:63-94

MILLÁN, R. -1945- Variaciones del zapallito amargo Cucurbita andreana y el origen de Cucurbita máxima. Revista Argentina de Agronomía 12: 86-93.

NEE, M. -1990- The domestication of Cucurbita (Cucurbitaceae). Economic Botany 44, Supplement: Bretting (ed.) New perspectives on the origin and evolution of New World domesticated plants.

NEWSOM, L. A., S. D. WEBB, \& J. S. DUNBAR -1993- History and geographic distribution of
Cucurbita pepo gourds in Florida. Journal of Ethnobiology 13(1): 75-98

PEARSALL, D. -1989- Paleoethnobotany, a handbook of procedures. Academic Press.

RATTO, N. M. ORGAZ \& L. COLL - 2010- Paisajes Agrícolas Prehispánicos en el oeste tinogasteño (Catamarca). XVII Congreso Nacional de Arqueología Argentina, T ${ }^{\circ}$ II, pp. 419-424. Mendoza.

RATTO, N., V. LEMA \& M. L. LÓPEZ - 2014Entierros y ofrendas: prácticas mortuorias, agrícolas y culinarias en los siglos XIII y XIV en Tinogasta (Catamarca, Argentina). Darwiniana. 2 (1):125-143

SANJUR, O. PIPERNO, D. ANDRES, T. \& SEL-BEAVER, L.-2002- Phylogenetic relationships among domesticated and wild species of Cucurbita (Cucurbitaceae) inferred from a mitochondrial gene: Implications for crop plant evolution and areas of origin. PNAS 99: 535-540.

STOTHERT, K., PIPERNO, D. \& ANDRES, T. - 2003- Terminal Pleistocene/Early Holocene human adaptation in coastal Ecuador: the Las Vegas evidence Quaternary International 109-110: 23-43

VALEGA ROSAS, R., ANDRES, T. \& NEE, M.-2004-The Goldman Cucurbit collecting Expedition in Perú. (informe).www.Cucurbita.org

WHITAKER, T.W. \& BEMIS, W.P. -1964- Evolution in the genus Cucurbita. Evolution 18 (4): 553-559.

WHITAKER, T.W. -1974-. Cucurbitales. Enciclopedia Britanica $15^{\circ}$ Ed. Helen Hemingway Benton Publised U.S.A

WHITAKER, T.W. \& BEMIS, W.P -1976Cucurbits.Cucumis, Citrullus, Cucurbita, La genaria (Cucurbitaceae).En N.W. Simmons (ED). Evolution of crop plant.pp:64-69 Longman Group Lted. London.NY 